\title{
Ambulatory Clinician's Guide to Inpatient Service: An Innovative Rapid Onboarding Strategy for the COVID-19 Pandemic
}

\author{
Richard L. Altman ${ }^{1} \quad$ Tyler Anstett ${ }^{2} \quad$ Jennifer R. Simpson ${ }^{3}$ Amira Del Pino-Jones ${ }^{2}$ Chen-Tan Lin ${ }^{1}$ \\ Jonathan Pell ${ }^{2}$ \\ ${ }^{1}$ Division of General Internal Medicine, University of Colorado School \\ of Medicine, Aurora, Colorado, United States \\ 2 Division of Hospital Medicine, University of Colorado School of \\ Medicine, Aurora, Colorado, United States \\ ${ }^{3}$ Department of Neurology, University of Colorado School of \\ Medicine, Aurora, Colorado, United States \\ Address for correspondence Richard L. Altman, MD, Division of \\ General Internal Medicine, University of Colorado School of Medicine, \\ 8th Floor, Academic Office 1, Mailstop B180, 12631 E 17th Avenue, \\ Aurora, CO 80045, United States \\ (e-mail: Richard.altman@cuanschutz.edu).
}

Appl Clin Inform 2020;11:802-806.

\begin{abstract}
Keywords

- COVID-19

- hospitalist

- training

- electronic health record

Background and Significance When hospitals are subject to prolonged surges in patients, such as during the coronavirus disease 2019 (COVID-19) pandemic, additional clinicians may be needed to care for the rapid increase of acutely ill patients. How might we quickly prepare a large number of ambulatory-based clinicians to care for hospitalized patients using the inpatient workflow of the electronic health record (EHR)?

Objectives The aim of the study is to create a successful training intervention which prepares ambulatory-based clinicians as they transition to inpatient services.

Methods We created a training guide with embedded videos that describes the workflow of an inpatient clinician. We delivered this intervention via an e-mail hyperlink, a static hyperlink inside of the EHR, and an on-demand hyperlink within the EHR.

Results In anticipation of the first peak of inpatients with COVID-19 in April 2020, the training manual was accessed 261 times by 167 unique users as clinicians anticipated being called into service. As our institution has not yet needed to deploy ambulatory-based clinicians for inpatient service, usage data of the training document is still pending.

Conclusion We intend that our novel implementation of a multimedia, highly accessible onboarding document with access from points inside and outside of the EHR will improve clinician performance and serve as a helpful example to other organizations during the COVID-19 pandemic and beyond.
\end{abstract}

\section{Background and Significance}

The coronavirus disease 2019 (COVID-19) pandemic has created a worldwide surge in acutely ill patients requiring inpatient medical care. ${ }^{1,2}$ Hospital systems are working quickly to increase their physical capacity to accommodate these patients even as they expand their frontline workforce of clinicians to care for them. ${ }^{3-5}$ The workforce supply

\section{received}

July 27, 2020

accepted after revision

September 23, 2020 problem is exacerbated by clinicians falling ill with the disease itself. ${ }^{6,7}$ The physician shortage has led to calls for assistance from medical professionals who do not currently practice inpatient medicine ${ }^{8}$ including ambulatory clinicians, procedural specialists, and retired physicians. Systems have been developed for the emergency credentialing and privileging of available medical professionals ${ }^{9}$ and national societies are working to match volunteer clinicians to
(C) 2020 Georg Thieme Verlag KG Stuttgart · New York
License terms

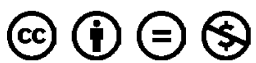


facilities where they are needed most. ${ }^{10}$ Unfortunately, hospital disaster management plans have traditionally focused on the organization of emergency and surgery personnel and not on the rapid training of a large hospitalist workforce. ${ }^{11}$ Thus, programs to rapidly train and support volunteer clinicians in the care and management of inpatients are needed but lacking. ${ }^{12}$

A significant challenge with any rapid inpatient clinician onboarding program is the complexity of the system-based practice of hospital medicine. Previous work pointing to the risks of introducing clinicians into new work environments has demonstrated that while clinicians may recognize the appropriate next steps to take in a clinical situation, they often lack the knowledge and skills necessary to take action in an unfamiliar environment. ${ }^{13}$ Likewise, attention that would normally be given to clinical care must be diverted to navigating otherwise routine workflows. ${ }^{13}$ In the absence of effective guidance, clinicians will resort to trial and error. ${ }^{13}$ However, when resource guides are readily available, clinicians demonstrate better adherence to standardized processes that can improve efficiency and effectiveness. ${ }^{14}$

One platform that inpatient clinicians use to support communication, clinical documentation, and clinical decision-making is the electronic health record (EHR). However, there are significant challenges in training large numbers of clinicians in a short period of time to both use the EHR in an inpatient setting and manage hospitalized patients. Historically, clinicians have found it difficult to absorb the details of a training all at once ${ }^{15}$ and have benefitted more from onthe-job support than pre-go-live training. ${ }^{16,17}$ Typically, the large-volume EHR onboarding that would occur during a hospital system go-live requires months of preparation and significant training resources. ${ }^{18,19}$

We believed that a training resource on the use of the inpatient EHR and associated workflows delivered within the EHR could solve these challenges. Our physician informatics team moved quickly to build an innovative training resource that is concise, scalable, easily adaptable, and available on demand. In doing so, we hoped to create a safer practice environment and reduce the stress experienced by ambulatory-based clinicians by better preparing them for inpatient service.

\section{Setting and Participants}

UCHealth University of Colorado Hospital is an academic tertiary care hospital in Aurora, Colorado. At baseline, it has 690 beds and is staffed by approximately 100 hospitalist clinicians dedicated to inpatient medicine and 50 clinicians who specialize in critical care medicine. To meet the anticipated demand for acute care during the COVID-19 outbreak, the hospital expanded its capacity to 928 beds while simultaneously increasing medical bed availability by cancelling elective surgeries.

The offices of 338 primary care clinicians in the UCHealth system are located within 20 miles of the main hospital. Clinicians specialize in general internal medicine (GIM), family medicine, geriatric medicine, or medicine-pediatrics. All ambulatory clinicians are trained on the same instance of the EHR (Epic Systems, Verona, Wisconsin), but most are trained only on the ambulatory module. In preparation for a possible transition to an inpatient role, primary care clinicians were surveyed about the last time they worked on an inpatient service, procedures they felt comfortable performing, and the level of patient acuity with which they felt comfortable.

\section{Methods}

To provide a core resource for the onboarding program, the project team (comprised of two clinician informaticists and four hospital-based providers) created a training manual that detailed the typical responsibilities of an inpatient clinician, including receiving sign-out, prerounding, rounding, billing, participating in multidisciplinary rounds, utilizing appropriate communication channels, and arranging admissions, transfers, and discharges (see - Supplementary Appendix A, available in the online version). The manual also described different clinical team structures, including those comprised only of a single physician and those that incorporate residents and/or advanced practice providers (e.g., nurse practitioners or physician assistants). Clinical advice regarding high-yield inpatient topics was included as were hyperlinks to COVID-19specific treatment information, which was still evolving.

When the training manual narrative described an inpatient workflow that took place solely in the EHR, a screenshot of the EHR was included to orient clinicians. Short (23 minutes) videos presenting live demonstrations of each major EHR workflow was embedded with links into the training manual to further explain the process. Each video could be paused and re-watched to facilitate learning at the pace and convenience of the individual clinician. All videos were scripted, recorded, and narrated by inpatient physician informaticists and produced in conjunction with the UCHealth information technology training team. The document and its contents were shared with nonauthor inpatient and outpatient clinicians during development for feedback on clarity and accuracy and to identify content gaps.

In addition to the training manual, an end-to-end demonstration of the inpatient workflow using the EHR was developed and delivered as a 45-minutes didactic session during a teleconferenced GIM grand rounds, which was video recorded and made available for on-demand viewing by volunteer clinicians.

The delivery systems for the training manual and the didactic session were unique. The training manual with links to associated videos were stored on a server that was securely accessible throughout the organization. Although a hyperlink to this document was e-mailed to clinicians with upcoming inpatient service dates, concerns were raised that clinicians might have trouble finding the hyperlink while on the inpatient service, which would limit the manual's benefit. Therefore, to better accommodate clinical workflow, hyperlinks to the manual were built within the EHR itself. These hyperlinks were accessible in the EHR in two ways: as a static hyperlink and an on-demand hyperlink. The static hyperlink was located on the common landing page of the EHR and when clicked, opened a new internet browser window outside of the EHR that displayed the document 


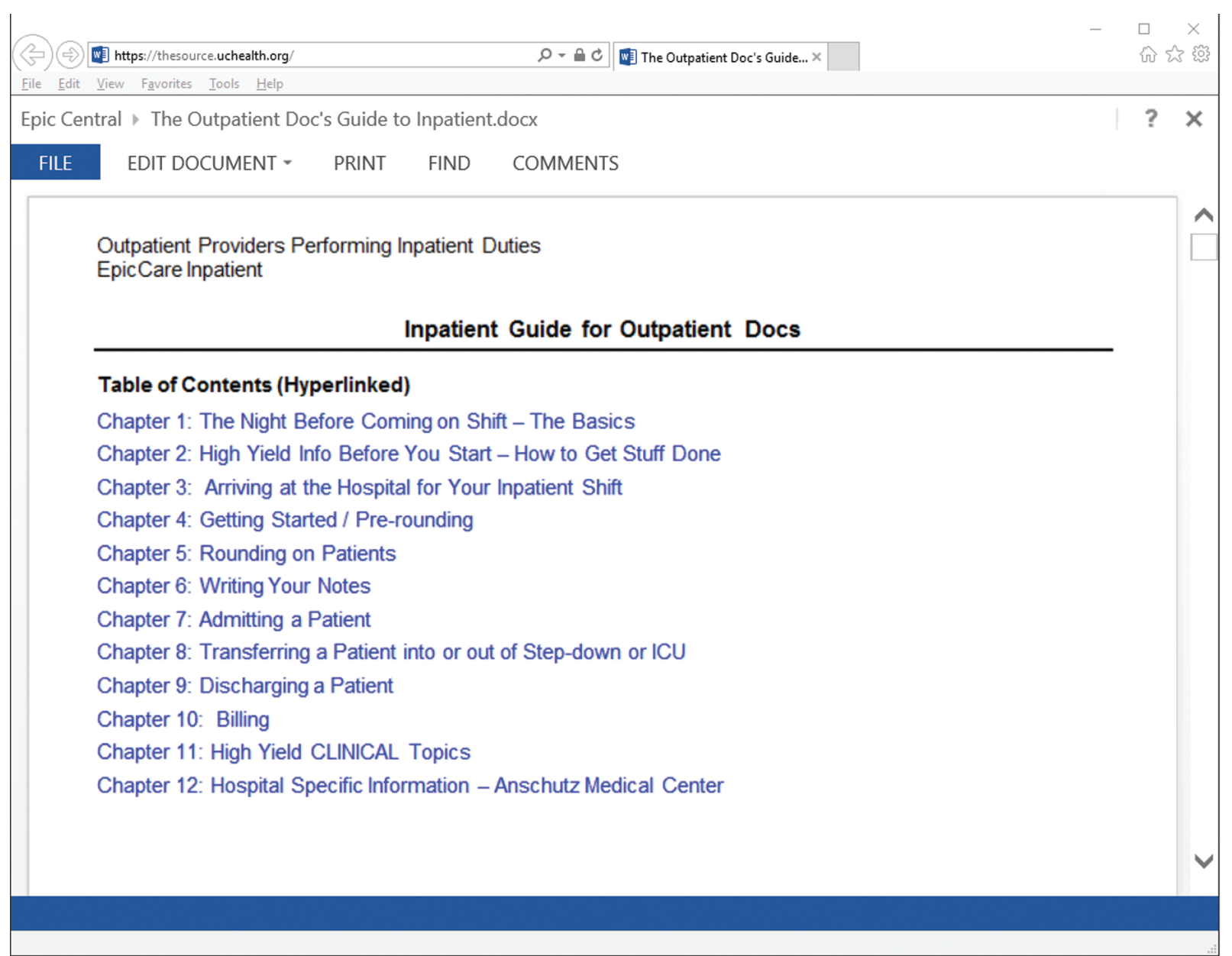

Fig. 1 The table of contents of the educational document as viewed from a pop-up browser window launched from inside the EHR. EHR, electronic health record.

(-Fig. 1). The on-demand hyperlink was summoned by the user from inside a patient note and opened the document within the EHR user interface itself ( - Fig. 2). Utilizing three different types of hyperlinks (e-mail, static, and on-demand) improved the accessibility of the training materials to clinicians in both the inpatient and outpatient settings. Further, because the hyperlink directed providers to a centrally stored document, the content of the document could be easily edited to reflect clinical and policy changes without disrupting clinicians' workflows in accessing the document.

\section{Results}

Our institution has been fortunate in that we have not yet needed to enlist the support of outpatient clinicians for inpatient service. Thus, the need for this tool has remained low and specific use metrics are unavailable. However, because our system prepared for the first peak of COVID-19 inpatients in April 2020, the training manual was accessed within our institution 261 times by 167 unique users as ambulatory-based clinicians anticipated being called into inpatient service. When shared on social media sites, the manual received over 1,000 views. Training manual access will be actively tracked as we anticipate the next wave of infections and hospitalizations.

\section{Discussion}

We built a multimedia, on-demand training module to rapidly prepare volunteer, ambulatory-based clinicians for inpatient service during the COVID-19 pandemic. This work is important given the expanded need for inpatient clinicians at many institutions and the desire for education stemming from ambulatory-based clinicians embarking on an unfamiliar workflow. Our innovation lies in the comprehensive delivery system of teleconferencing education, e-mail hyperlinks, workflow-appropriate static hyperlinks, and on-demand hyperlinks available throughout the EHR. Combined with our multimedia onboarding manual which utilizes text, pictures, and embedded videos, this project is novel and valuable. During a period of uncertainty in which there was an urgent need for information, this approach enabled our institution to alert clinicians to the presence of the training materials and allowed them highly-available access to these documents before, and importantly, during their service on an as-needed basis.

There was concern that information could quickly become outdated given the fluidity of the situation. ${ }^{20}$ Using hyperlinks pointed at the training document allowed us to update the information rapidly and seamlessly as needed in a central 


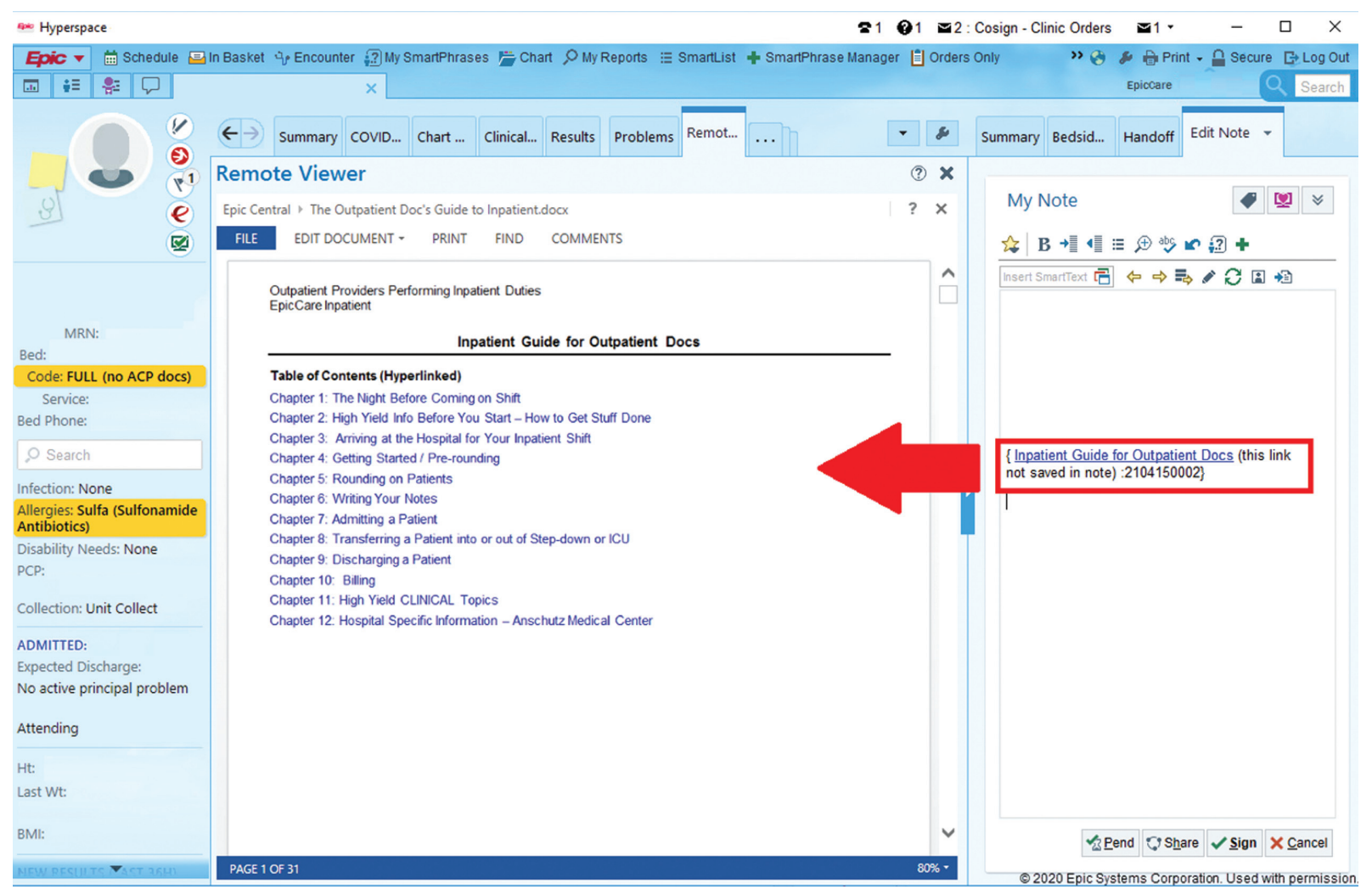

Fig. 2 The table of contents of the educational document as viewed in the EHR from an on-demand hyperlink (red square) summoned by the user from inside a patient note. In this example, the note is otherwise blank. The red arrow indicates that the educational document was triggered by the hyperlink.

location without disturbing access or needing to deliver modified versions. This ensured that material delivered to clinicians was always up to date and allowed us to quickly fill any identified informational gaps.

Our project has several limitations. The training materials were developed and deployed for use at a single hospital. The content will need to be adapted to specific workflows and the needs of outpatient physicians at other hospitals within our system. Hospitals external to our system using our training materials as templates may additionally have to adjust the content to reflect different EHR implementations. While initial clinician feedback has been very positive, there has been no formal usage data or end user feedback collected at this point. We anticipate tracking usage statistics and adjusting the tools as the pandemic evolves.

\section{Conclusion}

Given an immediate and unexpected need, our group was able to produce an innovative, comprehensive training document and implement strategic mechanisms of delivery to a targeted audience in a very short period of time. We intend that this novel, efficient, accessible, and practical method of preparing ambulatory-based physicians to assume inpatient responsibilities will ultimately improve clinical care and clinician performance while also reducing clinician stress levels. Although developed for an acute circumstance, lessons from this implementation may also lead to reexamination of routine on-boarding practices. We hope that these tools will provide a helpful foundation for our future projects and to other organizations during the COVID-19 pandemic and other prolonged surges in patient volumes.

\section{Clinical Relevance Statement}

As the coronavirus disease 2019 pandemic progresses, outpatient clinicians will be needed to supplement inpatient team but they may lack training regarding inpatient workflow and information system use. This article describes how a comprehensive, multimedia help document, accessible at multiple points in the workflow, was created to facilitate clinician training either in advance of service or "just-intime."

\section{Multiple Choice Questions}

1. When presented with an unclear path in an unfamiliar workflow, clinicians are most likely to ${ }^{13}$ :

a. Call a helpline for guidance.

b. Resort to trial and error.

c. Ask a colleague more familiar with the workflow for advice.

d. Ask nonclinical staff for help.

Correct Answer: The correct answer is option b. 
2. When presenting information to clinicians, the best course is to make the information available through: a. Multiple avenues pointing to a single source. b. A single avenue pointing to multiple sources. c. Multiple avenues pointing to multiple sources. d. A single avenue pointing to a single source.

Correct Answer: The correct answer is option a.

\section{Protection of Human and Animal Subjects}

Human and animal subjects were not included in this project.

\section{Conflict of Interest}

None declared.

\section{Acknowledgments}

The authors thank Esther Langmack, MD, for her editorial assistance and Kasey Bowden, MSN, FNP, AG/ACNP for her contributions to the training guide.

\section{References}

1 Paterlini M. On the front lines of coronavirus: the Italian response to covid-19. BMJ 2020;368:m1065

2 Hunter DJ. Covid-19 and the stiff upper lip-the pandemic response in the United Kingdom. N Engl J Med 2020;382(16):e31

3 Hick JL, Biddinger PD. Novel coronavirus and old lessons-preparing the health system for the pandemic. N Engl J Med 2020;382 (20):e55

4 Young S, Eisenberg A. New York's health care workforce braces for influx of retirees, inexperienced staffers. Available at: https:// www.politico.com/states/new-york/albany/story/2020/03/26/ new-yorks-health-care-workforce-braces-for-influx-of-retireesinexperienced-staffers-1269152. Internet. Politico; Apr 14, 2020 Accessed March 26, 2020

5 Lin C-T, Bookman K, Sieja A, et al. Clinical informatics accelerates health system adaptation to the COVID-19 pandemic: examples from Colorado. J Am Med Inform Assoc 2020. Doi: 10.1093/jamia/ocaa171

6 Wang D, Hu B, Hu C, et al. Clinical characteristics of 138 hospitalized patients with 2019 novel coronavirus-infected pneumonia in Wuhan, China. JAMA 2020;323(11):1061-1069
7 Chu J, Yang N, Wei Y, et al. Clinical characteristics of 54 medical staff with COVID-19: a retrospective study in a single center in Wuhan, China. J Med Virol 2020;92(07):807-813

8 de Blasio B. We Need All Medical Workers on the Front Lines. Internet. The New York Times; Apr 11, 2020. Available at: https:// www.nytimes.com/2020/04/03/opinion/coronavirus-deblasionyc.html. Accessed April 3, 2020

9 COVID-19 Emergency Declaration Blanket Waivers for Health Care Providers. Internet. Centers for Medicare \& Medicaid Services; Apr 14, 2020. Available at: https://www.cms.gov/files/document/covid19-emergency-declaration-healthcare-providers-fact-sheet.pdf. Accessed March 30, 2020

10 COVID-19 volunteer guide for health care professionals. Internet. American Medical Association; Apr 11, 2020. Available at: https:// www.ama-assn.org/delivering-care/public-health/covid-19-volunteer-guide-health-care-professionals. Accessed April 10, 2020

11 Persoff J, Ornoff D, Little C. The role of hospital medicine in emergency preparedness: a framework for hospitalist leadership in disaster preparedness, response, and recovery. J Hosp Med 2018;13(10):713-718

12 Cram P, Anderson ML, Shaughnessy EE. All hands on deck: learning to "un-specialize" in the COVID-19 pandemic. J Hosp Med 2020;15(05):314-315

13 Haas S, Gawande A, Reynolds ME. The risks to patient safety from health system expansions. JAMA 2018;319(17):1765-1766

14 Arriaga AF, Bader AM, Wong JM, et al. Simulation-based trial of surgical-crisis checklists. N Engl J Med 2013;368(03):246-253

15 Hill HK, Stewart DC, Ash JS. The training and support needs of faculty and students using a health information technology system were significant: a case study in a dental school. AMIA Annu Symp Proc 2010;2010:301-305

16 Ash JS, Stavri PZ, Kuperman GJ. A consensus statement on considerations for a successful CPOE implementation. J Am Med Inform Assoc 2003;10(03):229-234

17 Sieja A, Markley K, Pell J, et al. Optimization sprints: improving clinician satisfaction and teamwork by rapidly reducing electronic health record burden. Mayo Clin Proc 2019;94(05):793-802

18 Pantaleoni JL, Stevens LA, Mailes ES, Goad BA, Longhurst CA. Successful physician training program for large scale EMR implementation. Appl Clin Inform 2015;6(01):80-95

19 Stevens LA, Pantaleoni JL, Longhurst CA. The value of clinical teachers for EMR implementations and conversions. Appl Clin Inform 2015;6(01):75-79

20 Grange ES, Neil EJ, Stoffel M, et al. Responding to COVID-19: the UW medicine information technology services experience. Appl Clin Inform 2020;11(02):265-275 